\title{
Mitigation of Crosstalk on the SDSL Upstream Transmission with Vector Equalization
}

\author{
Liang C. Chu Martin Brooke \\ School of Electrical and Computer Engineering \\ Georgia Institute of Technology \\ Atlanta, GA 30332
}

\begin{abstract}
This paper provides a new approach to mitigate the crosstalk interference in the symmetric digital subscriber line (SDSL) upstream transmission. A vector channel equalizer has been proposed for the SDSL upstream receiver to enhance spectral compatibility for the SDSL services. This approach outperforms a conventional scalar equalizer, which used in the current SDSL systems, is about a $15 \mathrm{~dB}$ gain in mean squared error (MSE). It significantly suppresses the crosstalk degradation and ensures the quality of service (QoS) on the SDSL transmission throughput. Moreover, the enhancement of the SDSL receiver can benefit on the extension of the SDSL deployment coverage range and also guarantees its promising data rates on increasing numbers of customer subscribers.
\end{abstract}

\section{Introduction}

Telephone access networks were originally constructed from twisted copper pairs carrying voiceband signals up to the $4 \mathrm{kHz}$ frequency band. A combination of the existing copper infrastructure and digital subscriber line (DSL) transmission technologies became an emerging universal broadband access method to deliver the multimedia services to the customers [1]. However, there is a major threat to this vision of the future in the crosstalk, which is introduced by the adjacent channels. This crosstalk effect is especially bad near the central office, where the wireline binder groups are concentrated. Such degradation can severely reduce the transmission rate; sometimes even significantly polluting the copper network.

SDSL systems are modulated with $2 \mathrm{~B} 1 \mathrm{Q}$, which is a baseband 4-level pulse amplitude modulation. These systems transmit the same bit rate in upstream and downstream directions in full duplex. Normally, the transceivers on the upstream end are co-located in the local exchange carrier (LEC) central office (CO). The upstream SDSL receivers suffer severely from its selfNEXT (near-end-crosstalk), which causes significant degradation on its data rates [2]. In this paper, we derive a vector equalization for the SDSL upstream receiver, which can combat its crosstalk effectively. The SDSL upstream transmission system is proposed as a multiple input and multiple output (MIMO) channel model. Our simulation results have shown more than a $15 \mathrm{~dB}$ MSE gain compared with a conventional scalar decision feedback equalizer (DFE) that is being used in the current products.

In section 2, we review the effectiveness with NEXT. In section 3, we propose a MIMO version of SDSL channel model and its analysis. In section 4, we present some simulation results and discussions. The conclusion is in section 5 .

\section{SDSL Self-NEXT Limits and Reviews}

Studies on the received signal-to-noise ratio (SNR) of 1168, 1552 and 2320 kbps SDSL were computed on effectiveness with common 24 self-NEXT disturbers [3]. In the SDSL transmission, the self-NEXT limits it to a 6 $\mathrm{dB}$ SNR margin with the channel probability of bit error being $10^{-7}$. The simulation results were based on a $2 \mathrm{~B} 1 \mathrm{Q}$ SDSL receiver, which uses a scalar DFE with optimal minimum mean squared error (MMSE) tap values that has 12 baud-spaced forward filter taps and 64 feedback taps. The simulated SNR margin for different 26-AWG loop lengths has been shown in Fig. 1, (extracted from [3]).

The twisted pair channels (TPC) has been studied and modeled in a number of papers [4], [5]. It is assumed that the attenuation transfer characteristic $|H(f)|^{2}$, of the channel can be modeled by:

$$
|H(f)|^{2}=e^{-\alpha \sqrt{f}}
$$

where $\alpha=k \frac{l}{l_{\text {ref }}}, l=$ length of the channel in $\mathrm{ft}, l_{\text {ref }}$ $=\mathrm{a}$ reference length, $k=\mathrm{a}$ constant of the physical channel, and $f=$ frequency in $\mathrm{kHz}$. The $p s d, P_{\text {rec }}(f)$ of the received signal is given by:

$$
P_{r e c}(f)=|H(f)|^{2} P_{x m i t}(f)
$$

where $P_{x m i t}(f)$ is the two-side $p s d$ of the transmitted signal. In this paper, we target on the NEXT, which is the dominant factor on limiting the communication capabilities of the SDSL channel. The far-end-crosstalk (FEXT) has some minor effects on the SDSL throughput as well but is ignored here for the simplification purpose. In general, the FEXT can easily be counted into our 
proposed model in the following section. Following with [6], the NEXT transfer function can be written as

$$
H_{N E X T}(f)=j 2 \pi \cdot \int_{0}^{d} C_{n}(x, f) e^{-2 \gamma(f) x} d x
$$

where $x$ is the distance from the disturbing source, $d$ is the length of the twisted cable, and $C_{n}(x, f)$ is the NEXT unbalance function at distance $x$ and frequency $f$.

The squared gain of the NEXT transfer function [7], $\left|H_{N E X T}(f)\right|^{2}$ can be modeled as

$$
\left|H_{N E X T}(f)\right|=\beta \cdot f^{1.5}
$$

where $\beta$ is a constant of the twisted pair cable, and varies from the cable to cable.

The SDSL transmission rate is directly proportional to its SNR, as the TPC Shannon capacity is a theoretical upper bound on the data rate that can be reliably transmitted [7], [8]. Our review on the TPC and NEXT has shown that the higher the SDSL transmission rate, the worse on the channel SNR. Meanwhile, the results of Fig. 1 also show that the crosstalk greatly degrades the SDSL transmission rate, thus limiting its deployment range. This kind of the degradation is especially bad on the upstream receivers at LEC side due to the concentration of the twisted pair binders group.

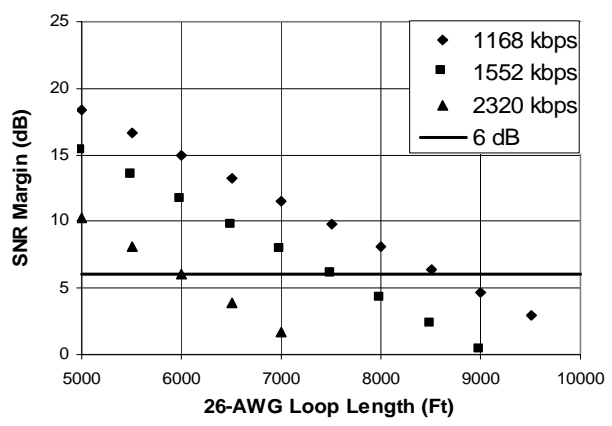

Fig. 1. Received SDSL SNR Margin with 24 Same-binder Self-NEXT

\section{SDSL Receiver Enhancement and Analysis}

A proposed MIMO SDSL upstream channel model has been introduced. We derive the vector equalization for the SDSL receiver, which can largely suppress the crosstalk degradation on the SDSL transmission throughput and greatly outperforms the current receiver design.

\subsection{Proposed Channel Model}

In a same binder group of a LEC at the CO side, the upstream SDSL transmission can be viewed as a multiple access channel. For example, two pairs of TPC can be shown in Fig. 2(a). The arrowed lines represent the upstream and downstream transmitted and NEXT signal flows. In general, this representation can be applied to any number of pairs of TPC in a same binder group. That is, an SDSL upstream receiver observes data from $n$ different sources through $m$ different linear coupling functions, resulting in an equivalent $n \times m$ MIMO channel model. The channel model is shown in Figure 2(b). The channel input $\boldsymbol{x}_{\boldsymbol{k}}$ is a sequence of vectors whose components represent the symbol sequences for each of $n$ SDSL transmitters. The channel transfer function is $\boldsymbol{H}(z)$ and background noise sequences are white Gaussian, expressed as $\boldsymbol{n}_{k}$. In most of the cases, the SDSL services are colocated. The channel response is most likely traced with a training signal. Some previous works have been studied on detection method for MIMO channels in various other applications [9], [10]. In this paper, we focus on the vector least mean-squared (LMS) algorithm for adaptation with training applied to MIMO SDSL channel model.

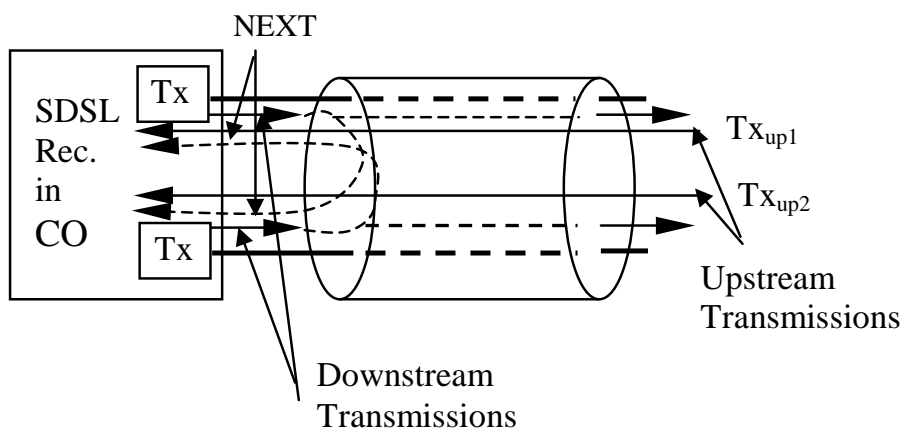

Fig.2 (a). Vector Multiaccess Channel in SDSL

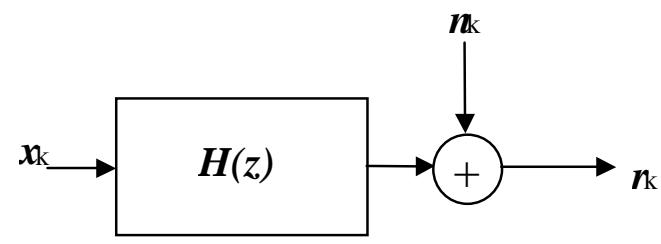

Fig.2 (b). MIMO SDSL Channel Model

\subsection{Vector Equalization for SDSL Systems}

A MIMO version of the least-mean square (LMS) approach is used to mitigate crosstalk on the SDSL system. The MIMO DFE [11] for the SDSL can be derived as following. It is a transversal filter with weight matrix $\mathbf{W}_{k}{ }^{T}=\left[\mathbf{W F}_{k}{ }^{T}, \mathbf{W B}_{k}{ }^{T}\right]$. There are $L+1$ matrix-valued taps on the feedforward described by the matrix $\mathbf{W F}_{k}{ }^{T}=\left[\mathbf{W F}_{0, k} \mathbf{W F}_{1, k} \ldots \mathbf{W F}_{L, k}\right]$ and $I$ matrix-valued taps on the feedback section as $\mathbf{W B}_{k}{ }^{T}=\left[\mathbf{W B}_{1, k} \mathbf{W B}_{2, k} \ldots \mathbf{W B}_{I, k}\right]$. Let $\mathbf{R}_{k}{ }^{T}=\left[\mathbf{r}_{k}{ }^{T} \mathbf{r}_{k-1}{ }^{T} \ldots \mathbf{r}_{k-(L+1)}{ }^{T}\right]$ be a vector of SDSL equalizer inputs and $\hat{\mathbf{X}}_{k}{ }^{T}=\left[\mathbf{x}_{k-1}{ }^{T} \mathbf{x}_{k-2}{ }^{T} \ldots \mathbf{x}_{k-I}{ }^{T}\right]$ is the previously detected symbols, so that the SDSL equalizer output at time $k$ is

$\mathbf{y}_{k}=\mathbf{W F}_{k}^{T} \mathbf{R}_{k}-\mathbf{W B}_{k}{ }^{T} \widehat{\mathbf{X}}_{k}$. 
The goal of the equalizer is to minimize the MSE cost function:

$$
\mathbf{J}=E\left[\left\|\mathbf{y}_{k}-\mathbf{x}_{k}\right\|^{2}\right]
$$

where $\boldsymbol{x}_{k}$ is the desired SDSL signal. The complex gradient [12] of Eq. (5) with respect to $\boldsymbol{W}_{\boldsymbol{k}}$ is:

$$
\nabla \mathbf{J}=2 E\left[\mathbf{V}_{k}^{*} \mathbf{e}_{k}^{T}\right]
$$

where $\mathbf{V}_{k}=\left[\mathbf{R}_{k}, \hat{\mathbf{X}}_{k}\right]$, and $\mathbf{e}_{k}=\mathbf{y}_{k}-\mathbf{x}_{k}$ is the error between the equalizer output and the desired SDSL signal, respectively. With the stochastic approximation of the gradient in the steepest descent algorithm on Eq. (6), the vector LMS algorithm on the SDSL DFE weighttap updating function is:

$$
\mathbf{W}_{k+1}=\mathbf{W}_{k}-\mu \mathbf{V}_{k}^{*} \mathbf{e}_{k}^{T}
$$

where $\mu$ is the step size.

By substituting $\mathbf{e}_{k}=\mathbf{W}_{k}^{T} \mathbf{V}_{k}-\mathbf{x}_{k}$ into Eq. (7), then:

$$
\nabla \mathbf{J}=2\left(\boldsymbol{\Phi} \mathbf{W}_{k}-\mathbf{P}\right)
$$

where $\boldsymbol{\Phi}=E\left[\mathbf{V}_{k}^{*} \mathbf{V}_{k}^{T}\right]$ and $\mathbf{P}=E\left[\mathbf{V}_{k}^{*} \mathbf{x}_{k}^{T}\right]$. It follows that the optimal weight-tap is given by:

$$
\mathbf{W}_{\text {opt }}=\boldsymbol{\Phi}^{-1} \mathbf{P}
$$

and the minimum mean squared error is:

$$
\xi_{\min }=E\left[\left\|\mathbf{x}_{k}\right\|^{2}\right]-\operatorname{tr}\left(\mathbf{P}^{H} \mathbf{W}_{\text {opt }}\right)
$$

where $\mathbf{P}^{H}$ is conjugate transpose of $\mathbf{P}$.

For the convergence of the vector LMS on the SDSL receiver, the vector LMS uses a time average to estimate an ensemble average, and the coefficient trajectories are random in nature. The rule on measuring performance is the rate of convergence of the average MSE. With the detailed approaches shown on [13], we can find the step size that maximizes the rate of decrease of the MSE is:

$$
\mu_{\text {opt. }}=\frac{1}{n L \lambda}
$$

where $n$ is the dimension of the vector, and $\lambda$ is the eigenvalue of the diagonal matrix $\Phi$. It is also known that the choice of the step size is a trade off between speed of convergence and excess asymptotic MSE.

\section{Example and Discussion}

Consider on any pairwise SDSL TPC crosstalk in a binder group. The proposed channel model as derived in section 3.1, which is shown in Fig. 2(b), with a channel transfer function as:

$$
\mathbf{H}(f)=\left[\begin{array}{cccc}
1 & H_{\text {NEXT }}(f) & 0 & H_{\text {NEXT }}(f) \\
0 & H_{N E X T}(f) & 1 & H_{N E X T}(f)
\end{array}\right]
$$

where $H_{\text {NEXT }}(f)$ is the NEXT coupling path function as shown in Eq. (3). In a binder group, the SDSL channels are statistically independent and symmetric. We assume having the same pairewise coupling path function, $H_{\text {NEXT }}(f)$, for neighboring adjacent pairs. Also assume that the SDSL upstream and downstream transmitters are transmitted with the independent baseband PAM signal in a binder group. In this example, the four transmitted signals are as $\mathbf{x}_{\mathbf{k}}=\left[x_{1 \text { up }}, x_{1 \text { down }}, x_{2 \text { up }}, x_{2 \text { down }}\right]^{T}$, where $x_{i u p}$ is the upstream transmitted signal and $x_{\text {idown }}$ is the downstream transmitted signal, $i=1,2$. The coupling path function $H_{N E X T}(f)$, is approximately 0.3 from Fig. (1) for $2320 \mathrm{kbps}$ SDSL service. That is

$$
\mathbf{r}_{\mathbf{k}}=\mathbf{H}(f) \cdot \mathbf{x}_{\mathbf{k}}=\left[\begin{array}{llll}
1 & 0.3 & 0 & 0.3 \\
0 & 0.3 & 1 & 0.3
\end{array}\right] \cdot\left[\begin{array}{c}
x_{1 u p} \\
x_{1 d o w n} \\
x_{2 u p} \\
x_{2 d o w n}
\end{array}\right]
$$

The Fig. 3(a) shows two scalar DFE receivers with the parallel conventional LMS algorithms. The Fig. 3(b) shows a MIMO DFE receiver employing the vector LMS algorithm.

A key issue that needs to be emphasized here is that the LMS algorithm of the vector equalizer is not equivalent to the parallel independent scalar equalizers. The vector LMS algorithm exploits the crosstalk between users, whereas a bank of scalar LMS algorithms treats the crosstalk as background noise. It is better to exploit the crosstalk than to treat it together with background noise. That is why the vector SDSL equalizer can significantly outperform the current scalar equalizer.

In Fig. 4, we show the simulation results of the MSE in $\mathrm{dB}$ on the $2320 \mathrm{kbps}$ pairwise SDSL system at $7000 \mathrm{ft}$, with the equalizers chosen from Fig. 3(a) \& (b). The step size was $\mu=0.06$ for two of the scalar equalizer and the step size for the vector equalizer was chosen as in Eq. (11), with $\mu_{\text {vector }}=0.036$. There was 20 forward taps and 84 feedback taps used for the vector equalization. It has been shown that the conventional SDSL receiver performs poorly on suppressing the crosstalk. The MSE for each user is about $-15 \mathrm{~dB}$, which results in a total of $-12 \mathrm{~dB}$. The vector SDSL receiver effectively mitigates the crosstalk and outperforms conventional equalizers more than $15 \mathrm{~dB}$ in MSE on the SDSL upstream transmission channel.

Based on this approach, the crosstalk for the SDSL services can be largely suppressed and the QoS on the SDSL transmission throughput has been ensured. With a reasonable prediction on this modified SDSL system for the deployment, its loop coverage range can be extended by more than 2000 feet and also increase the numbers of customer subscribers at a lower economic cost. 


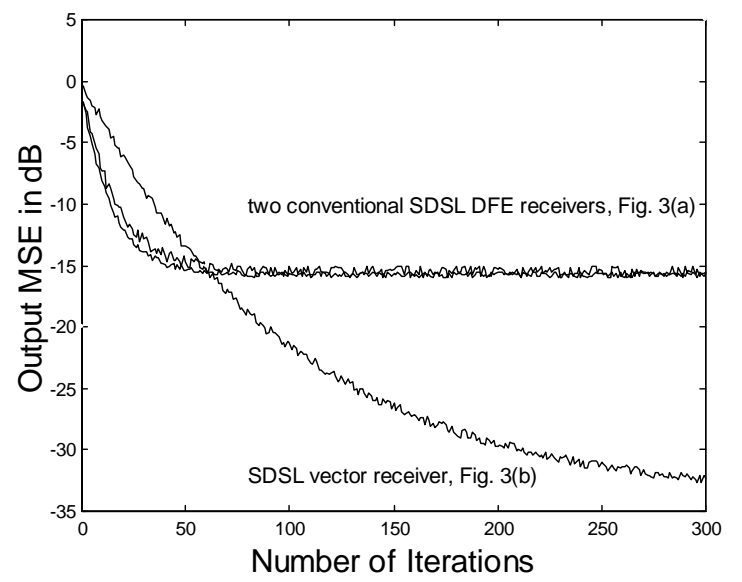

Fig. 4. Output MSE for the Scalar and Vector SDSL Receivers

\section{Conclusion}

The proposed vector DFE SDSL receiver can suppress the channel crosstalk significantly. This enhancement of the SDSL upstream receiver makes the capacity of the SDSL network utilized much better. With this modification on the SDSL receiver, the computational complexity is increased about $35 \%$ and its latency is approximately $10 \mathrm{~ms}$. Our goal on this study is to allow the SDSL network supporting the fast Internet access service with its sub-optimal capacity, and extends the SDSL deployment range to additional $2000 \mathrm{ft}$ or more with QoS on customer promising throughput.

\section{References:}

[1] Young, G., Forster K. T., and Cook J. W.: "Broadband multimedia delivery over copper," BT Tech. J., Vol. 13, No. 4, October 1995.

[2] Kliger, Avi: "Spectral compatibility of multi rate SDSL systems," T1E1.4/99-048, February 1999.

[3] Valenti, Craig: "Regarding the spectral compatibility of 2B1Q SDSL," T1E1.4/99-261, June 1999.

[4] Cox, S. A., and Adams, P. F., "Analysis of Digital Transmission Techniques for a Local Network," Brit. Telecommun. Technol. J., Vol. 3, No.3, July 1985.

[5] Conte, R. A., “ A Crosstalk Model for Balanced Digital Transmission in Multipair Cables," AT\&T Tech. J., Vol. 65, No. 3, May-June, 1986.

[6] Gibbs, A. J. and Addie, R., "The Covariance of the Near End Crosstalk and its Application to PCM System Engineering in Multipair Cable," IEEE Trans. Commun., Vol. COM-27, February 1979.

[7] Kalet, I and Shamai, S., "On the Capacity of a TwistedWire Pair: Gauaaian Model,” IEEE Trans. Commun., Vol. 38, March 1990.

[8] Cover, T., and Thomas, J., Elements of Information Theory. Wiley, New York, 1991.

[9] Duel-Hallen, A., "Equalizers for multipleinput/multiple-output channels and PAM systems with cyclostationary input sequences," IEEE Journal on Selected Areas in Commun.," 10:3, pp. 630-639, April, 1992.

[10] Verdu, S., "Recent progress in multiuser detection," Advances in Communications and Signal Processing, Sprnger-Verlag, New York, 1989.

[11] Widrow, B. and Stearns, S. D.: "Adaptive signal processing," Prentic-Hall, New Jersey, 1985.

[12] Haykin, S.: “Adaptive Filter Theory," Second Edition, Prentic-Hall, New Jersey, 1991.

[13] Lee, E. A. and Messerschmitt, D. G., Digital Communications, Second Edition, Kluwer, Boston, 1994. 

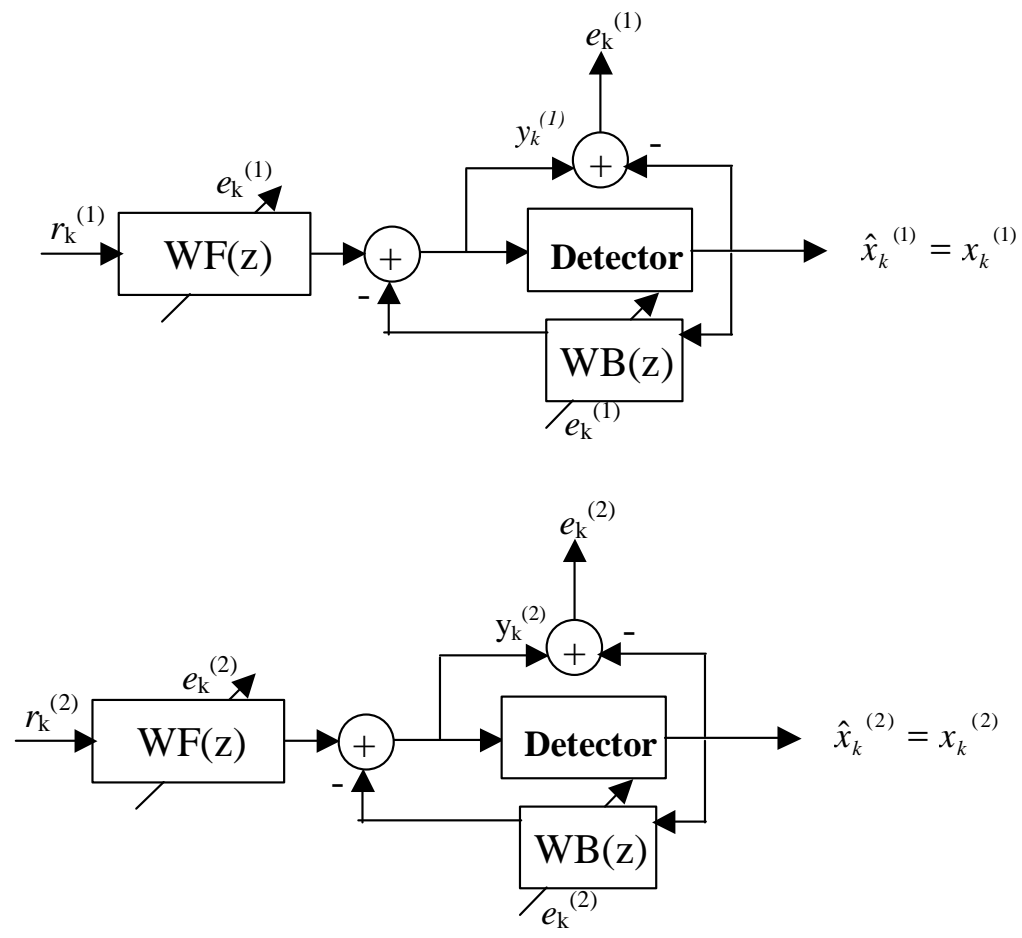

Fig. 3(a). Conventional Scalar DFE Receiver

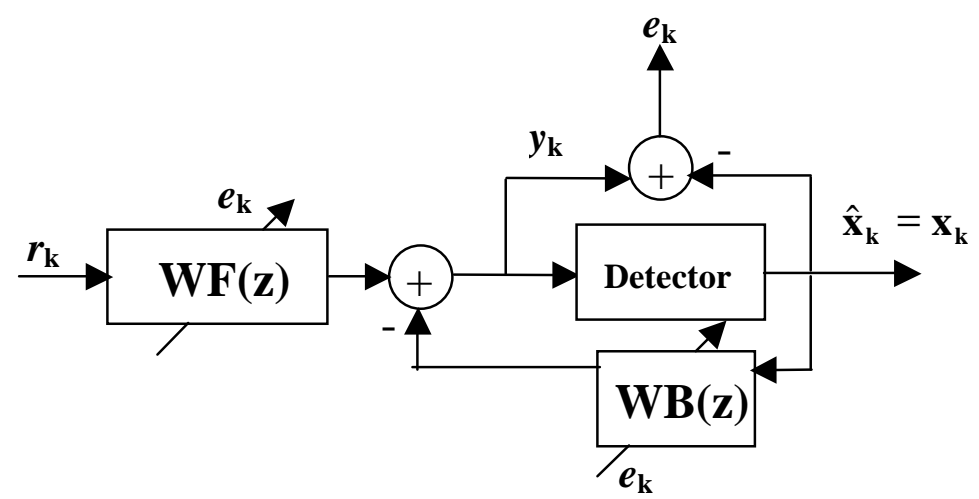

Fig. 3(b). Vector DFE Receiver 